ORIGINAL RESEARCH

\title{
Ginkgo biloba Decreases Acute Mountain Sickness in People Ascending to High Altitude at Ollagüe (3696 m) in Northern Chile
}

Fernando A. Moraga, BSc, MSc; Alejandro Flores, MD; Jordi Serra, MD; Carla Esnaola, MD; Corina Barriento, MD

From the Laboratorio de Fisiología, Escuela de Medicina, Universidad Católica del Norte, Coquimbo-Chile.

\begin{abstract}
Objective.-To determine the prophylactic effect of Ginkgo biloba (doses $80 \mathrm{mg} / 12 \mathrm{~h}, 24 \mathrm{~h}$ before high-altitude ascension and with continued treatment) in preventing acute mountain sickness (AMS) at $3696 \mathrm{~m}$ in participants without high-altitude experience.

Methods.- Thirty-six participants who reside at sea level were transported to an altitude of 3696 $\mathrm{m}$ (Ollagüe). The participants were divided into 3 groups and received $G$ biloba $(n=12) 80 \mathrm{mg} / 12$ h, acetazolamide $(n=12) 250 \mathrm{mg} / 12 \mathrm{~h}$, or placebo $(n=12) 24$ hours before ascending and during their 3-day stay at high altitude. The Lake Louise Questionnaire constituted the primary outcome measurement at sea level and at $3696 \mathrm{~m}$. A Lake Louise Self-Report Score greater than 3 was indicative of AMS. Oxygen saturation, heart rate, and arterial pressure were taken with each evaluation for AMS.

Results.-A significant reduction in AMS was observed in the group that received $G$ biloba (0\%, $P<.05)$ comparison with the groups receiving acetazolamide $(36 \%, P<.05)$ or placebo $(54 \%)$. No difference was observed in arterial oxygen saturation in the $G$ biloba $(92 \pm 2)$ vs the acetazolamide $(89 \pm 2)$ groups. However, a marked increased saturation in arterial oxygen was seen in comparison with the placebo group $(84 \pm 3, P<.05$ ). No statistically significant differences were observed in mean arterial pressure or heart rate.

Conclusions.-This study provides evidence supporting the use of $G$ biloba in the prevention of AMS, demonstrating that 24 hours of pretreatment with $G$ biloba and subsequent maintenance during exposure to high altitude are sufficient to reduce the incidence of AMS in participants with no previous high-altitude experience.
\end{abstract}

Key words: Ginkgo biloba, acute mountain sickness, high altitude, prophylaxis, Lake Louise questionnaire

\section{Introduction}

The first documented account in the western hemisphere of malaise associated with high-altitude ascension was made by a priest named Jose de Acosta in 1590 during a Hispanic conquest in South America. While crossing the Andean mountain range in Perú (Pariacaca) at 4800 $\mathrm{m}$, he described a characteristic malaise in animals and human beings due to the thinness of the atmosphere. ${ }^{1}$ However, a more complete description of "soroche" (acute mountain sickness [AMS]) was compiled by Rav-

Corresponding author: Fernando A. Moraga, BSc, MSc, Laboratorio de Fisiología, Escuela de Medicina, Universidad Católica del Norte, PO Box 117, Coquimbo, Chile (e-mail: fmoraga@ucn.cl). enhill in $1913 .^{2}$ Presently, AMS in adults is characterized by the following signs and symptoms: headache, fatigue and/or weakness, gastrointestinal disorders, dizziness/lightheadedness, difficulty in sleeping, etc. Symptoms can appear between 4 and 8 hours and 96 hours after arrival at high altitude. ${ }^{3}$

Annually, millions of people visit areas of high altitude for a variety of purposes: tourism, sports, jobs, and/ or military operations. ${ }^{3-5}$ Several instruments or questionnaires have been developed to assess the symptoms and signs of AMS, and these questionnaires are principally used among trekkers, climbers, and tourists. ${ }^{3}$ The likelihood of developing AMS is determined by individual susceptibility, speed of ascension, altitude attained, 
previous acclimatization, age, and gender. ${ }^{3,4}$ A $60 \%$ incidence of AMS was reported in people having no highaltitude experience in Chungará at $4250 \mathrm{~m}^{5}$ and in climbers that flew from sea level to $2800 \mathrm{~m}$ at Pheriche before climbing to $4243 \mathrm{~m}^{3}$

The pathophysiology of AMS is not totally understood, but it is apparent that administration of prophylactic treatments is helpful. Historically, acetazolamide has been used as a gold standard in treatments to prevent malaise symptoms at high altitudes. Acetazolamide is a diuretic that inhibits carbonic anhydrase, producing an increase in renal secretion of potassium and bicarbonate. The aim of prophylactic treatment is to induce metabolic acidosis, thereby maintaining hyperventilation at high altitude and enhancing systemic oxygenation, which decreases the symptoms of AMS. ${ }^{6}$ In the past, doses of 1 to $2 \mathrm{~g} / \mathrm{d}$ were used to prevent high altitude sickness, but more recently, doses of $250 \mathrm{mg} / 12 \mathrm{~h}$ or $125 \mathrm{mg} / 8 \mathrm{~h}$ have been found sufficient to reduce the symptoms of AMS. ${ }^{7}$

New protocols involving natural hypotensor administration, such as chachacoma (Senecio graveolens w.) tea ${ }^{8}$ and coca leaf chew, ${ }^{9}$ have been tried to reduce AMS symptoms. However, these studies do not demonstrate a decrease in AMS symptoms. Prophylactic antioxidant vitamin supplementation has also been tried to prevent AMS. ${ }^{10}$ In addition, Ginkgo biloba leaves have a potent antioxidant effect and induce arterial vasodilation, suggesting a relation with nitric oxide (NO) and a remarkable potential in hemodynamic disorders decreasing free radicals produced during exposure to hypoxia. ${ }^{11,12}$ Furthermore, studies in humans exposed to a hypoxic condition showed that $G$ biloba administered for 5 days decreased exhaled nasal NO, indicating an effect on $\mathrm{NO}$ metabolism. ${ }^{13}$ In a study in which rats were exposed to simulated hypobaric hypoxia, treatment with $G$ biloba prevented high-altitude pulmonary edema. ${ }^{14}$ However, few studies in humans have been performed to evaluate the prophylactic role of $G$ biloba in preventing AMS. The first studies were performed by Roncin and colleagues ${ }^{15}$ in climbers at $5400 \mathrm{~m}$, in which a group receiving $G$ biloba showed no symptoms of cerebral AMS and respiratory AMS was only present in $13.6 \%$ of the participants vs $82 \%$ of the control group. In a rapid ascent study to $4205 \mathrm{~m}$, a 24-hour pretreatment of $G$ biloba $(180 \mathrm{mg} / \mathrm{d})$ before climbing reduced the incidence of AMS. ${ }^{16}$ On the other hand, a randomized study showed that $G$ biloba was not effective at preventing AMS in trekking up to 4280 $\mathrm{m}$, when its use was initiated at this altitude and continued up to $4928 \mathrm{~m} .{ }^{17} \mathrm{In}$ addition, a 24 -hour pretreatment before ascending to $3800 \mathrm{~m}$ did not prevent the symptoms of AMS. ${ }^{18}$ These controversial results suggest that treatment with $G$ biloba requires a pretreatment period for effectively preventing AMS.
Our aim was to evaluate prophylactic treatment using G biloba $(80 \mathrm{mg} / 12$ h) 24 hours before ascending to an altitude of $3696 \mathrm{~m}$ with continued treatment for 3 days in order to reduce the symptoms of AMS in a population without experience in high-altitude ascension.

\section{Participants, materials, and methods}

\section{PARTICIPANTS}

Fifty students from the Medical College at the University of Antofagasta voluntarily consented to participate in the present study. Thirteen students were excluded for having previous experience with high altitude $(1500+$ $\mathrm{m})$. Thirty-eight students were then evaluated by physicians, and 2 of them were excluded for having incidents of seizure and recent pneumonia, leaving 36 students who signed consent to participate in this study. This study was done according to the Helsinki guidelines and those of the Ethical Committee at the University of Antofagasta.

The 36 participants (male) were separated at random into 3 groups, giving a number for each participant, and the randomization was computer generated: the first group $(n=12)$ received $G$ biloba extract EGb761 (Rokan, Andromaco Laboratories, Chile) given in doses of $80 \mathrm{mg} / 12 \mathrm{~h}$ and having a composition of $24 \%$ Ginkgo flavone glycosides and 6\% terpenes lactones; the second group $(n=12)$ received acetazolamide in doses of 250 $\mathrm{mg} / 12 \mathrm{~h}$; and the third group $(n=12)$ received placebo.

\section{EXPERIMENTAL PROTOCOL}

Each group was evaluated under 2 conditions: 1) at sea level (Antofagasta), where a month before ascending to high altitude participants received placebo, acetazolamide, or $G$ biloba for 3 days; and 2) at high altitude (Ollagüe town, $3969 \mathrm{~m}$ ), where the same participants received placebo, acetazolamide, or G biloba 24 hours before ascending and continued for 3 days. Acute mountain sickness symptoms were evaluated in both conditions using questionnaires that assessed 5 items: headache, gastrointestinal symptoms, fatigue and/or weakness, dizziness/lightheadedness, and difficulty in sleeping. Each item was scored from 0 to 3 . We considered AMS to be manifested in participants having a total score $\geq 3$ or having scored 1 symptom with a value of $3^{19}$; Cardiorespiratory variables were evaluated as well. Oxygen saturation $\left(\mathrm{SpO}_{2}\right)$ and heart rate $\left(\mathrm{min}^{-1}\right)$ were measured by means of a Nonin $8500 \mathrm{M}$ pulse oxymeter (Nonin Medical Inc, Plymouth, MN), and arterial pressure was evaluated by using a mercury column sphygmomanometer. These cardiorespiratory parameters were 
Table 1. Characteristics of the study groups*

\begin{tabular}{llllll}
\hline & $\mathrm{n}$ & Age $(\mathrm{y})$ & Weight $(\mathrm{kg})$ & Height $(\mathrm{m})$ & $C M I\left(\mathrm{~kg} / \mathrm{m}^{2}\right) \dagger$ \\
\hline Placebo & 12 & $22.2 \pm 1.1$ & $72.4 \pm 10.1$ & $1.71 \pm 0.1$ & $24.8 \pm 4.9$ \\
Acetazolamide & 12 & $23.3 \pm 1.2$ & $73.9 \pm 12$ & $1.70 \pm 0.1$ & $25.6 \pm 4.8$ \\
Ginkgo biloba & 12 & $22.1 \pm 2.9$ & $74.3 \pm 11.2$ & $1.74 \pm 0.1$ & $24.5 \pm 4.4$ \\
\hline
\end{tabular}

$*$ Mean $\pm \mathrm{SD}$; no significant differences were observed at sea level.

$\dagger \mathrm{CMI}$ indicates corporal mass index.

taken simultaneously with each evaluation for AMS at sea level and high altitude at 8:00 AM in the morning for 3 days.

\section{ASCENSION TO OLLAGÜE}

Ascension to Ollagüe $(3696 \mathrm{~m})$ began at 8:30 AM from Antofagasta (sea level) via highway. Arrival to Calama (2400 m) at 12:30 PM was followed by a 1-hour stop, and arrival at Ollagüe was at 5:00 PM. Travel time was approximately 8.5 hours.

\section{STATISTICAL ANALYSIS}

The incidence of AMS was expressed as a percentage, and the differences among groups were tested by ratio comparison; $P<.05$ was considered significant. The cardiorespiratory values, AMS score, and each symptom score were expressed as mean \pm SD. The difference among groups was evaluated using analysis of variance followed by rank analysis with Newman-Keuls test, where $P<.05$ was considered significant (Statistica, Statsoft, Tulsa, OK).

\section{Results}

\section{SEA LEVEL EVALUATIONS}

Table 1 summarizes physical parameters (eg, age, weight, height, corporal mass index) for each of the 3 groups; no statistically significant differences were observed between these groups. Moreover, treatment had no effect on AMS symptoms or cardiorespiratory variables during the baseline measures in any of the groups (Table 2).

\section{EVALUATIONS AT HIGH ALTITUDE}

Table 2 shows the AMS scores for each of the participants at sea level and high altitude during the 3 days of treatment. At sea level, AMS values were stable and no significant differences were seen during the 3 days of treatment in any of the groups. At high altitude, there was an increase in the AMS scores in the placebo and acetazolamide groups, whereas no change in the AMS scores was observed in the $G$ biloba group. There was little variation in the AMS scores during the 3 days at high altitude for all 3 groups.

Table 3 lists the averages for AMS scores during 3 days at sea level and high altitude for each group. At sea level, no significant differences were observed in AMS symptoms in any of the groups. However, a significant increase in headaches and difficulty in sleeping was observed in the placebo and acetazolamide groups at high altitude vs sea level. In contrast, no changes in any symptoms were observed in the $G$ biloba group. The incidence of AMS observed in the group treated with $G$ biloba was $0 \%$ during 3 days, which was significantly lower than the other groups treated with acetazolamide or placebo. On the other hand, acetazolamide decreased the incidence of AMS to $36 \%$, and this was significantly lower than the placebo group that presented an incidence of $56 \%$ at $3696 \mathrm{~m}$.

Table 4 summarizes the mean for cardiorespiratory responses for each group at sea level and high altitude for 3 days. High-altitude exposure induced an increase in the heart rate and a decrease in the arterial oxygen saturation in all groups $(P<.05)$, whereas no changes were observed in the systolic and diastolic arterial pressures. Prophylactic treatment with $G$ biloba or acetazolamide significantly increased arterial oxygen saturation compared with the placebo group. However, no differences were detected in heart rate or systolic and diastolic arterial pressures.

\section{Discussion}

Our study suggests that a 24-hour pretreatment with $G$ biloba before ascending to high altitudes and its continued administration while at high altitudes decreases the incidence of AMS and induces an increase in oxygen saturation in participants with no climbing experience. These findings corroborate and support the prophylactic use of $G$ biloba in the prevention of AMS. ${ }^{15,16,20,21}$

Our results are in contrast with other studies, in which 
Table 2. Acute mountain sickness scores at sea level and high altitude

\begin{tabular}{|c|c|c|c|c|c|c|c|c|c|}
\hline & \multicolumn{3}{|c|}{ Placebo } & \multicolumn{3}{|c|}{ Acetazolamide } & \multicolumn{3}{|c|}{ Ginkgo biloba } \\
\hline & Day 1 & Day 2 & Day 3 & Day 1 & Day 2 & Day 3 & Day 1 & Day 2 & Day 3 \\
\hline \multicolumn{10}{|c|}{ Participant at sea level } \\
\hline 1 & 1 & 1 & 1 & 1 & 1 & 1 & 1 & 1 & 1 \\
\hline 2 & 0 & 0 & 1 & 0 & 1 & 1 & 1 & 0 & 0 \\
\hline 3 & 0 & 1 & 1 & 1 & 0 & 1 & 0 & 1 & 1 \\
\hline 4 & 0 & 1 & 0 & 1 & 0 & 1 & 0 & 0 & 1 \\
\hline 5 & 0 & 1 & 0 & 1 & 0 & 0 & 1 & 0 & 0 \\
\hline 6 & 2 & 1 & 1 & 1 & 1 & 1 & 2 & 1 & 2 \\
\hline 7 & 1 & 1 & 0 & 1 & 1 & 1 & 1 & 1 & 0 \\
\hline 8 & 0 & 0 & 1 & 1 & 1 & 1 & 0 & 1 & 1 \\
\hline 9 & 1 & 1 & 1 & 1 & 2 & 1 & 1 & 1 & 1 \\
\hline 10 & 2 & 1 & 1 & 0 & 0 & 1 & 0 & 1 & 1 \\
\hline 11 & 1 & 1 & 1 & 1 & 0 & 0 & 2 & 1 & 1 \\
\hline 12 & 1 & 0 & 1 & 1 & 1 & 1 & 1 & 1 & 1 \\
\hline Mean & 0.75 & 0.75 & 0.75 & 0.83 & 0.76 & 0.83 & 0.83 & 0.75 & 0.83 \\
\hline SD & 0.75 & 0.45 & 0.45 & 0.39 & 0.65 & 0.39 & 0.72 & 0.45 & 0.58 \\
\hline \multicolumn{10}{|c|}{ Participant at high altitude } \\
\hline 1 & 10 & 7 & 5 & 2 & 2 & 2 & 0 & 0 & 0 \\
\hline 2 & 2 & 2 & 2 & 2 & 2 & 2 & 1 & 1 & 1 \\
\hline 3 & 7 & 6 & 4 & 7 & 3 & 3 & 0 & 0 & 0 \\
\hline 4 & 5 & 4 & 2 & 2 & 1 & 1 & 2 & 1 & 0 \\
\hline 5 & 6 & 5 & 4 & 2 & 1 & 1 & 1 & 0 & 1 \\
\hline 6 & 2 & 2 & 2 & 2 & 2 & 2 & 2 & 2 & 1 \\
\hline 7 & 2 & 2 & 2 & 6 & 4 & 3 & 0 & 1 & 1 \\
\hline 8 & 5 & 2 & 2 & 6 & 4 & 3 & 1 & 1 & 0 \\
\hline 9 & 5 & 7 & 5 & 3 & 3 & 2 & 2 & 2 & 1 \\
\hline 10 & 3 & 5 & 5 & 2 & 1 & 1 & 2 & 2 & 2 \\
\hline 11 & 2 & 2 & 2 & 4 & 1 & 1 & 0 & 0 & 1 \\
\hline 12 & 2 & 2 & 2 & 4 & 1 & 1 & 0 & 0 & 1 \\
\hline Mean & 4.33 & 3.83 & 3.08 & 3.42 & 2.08 & 1.92 & 1.08 & 0.92 & 0.83 \\
\hline SD & 2.5 & 2.08 & 1.38 & 1.93 & 1.16 & 0.90 & 0.90 & 0.79 & 0.58 \\
\hline
\end{tabular}

$G$ biloba did not reduce AMS. These disparities could be explained because of experimental design differences, starting treatment 24 hours before hypoxia exposure, total time of treatment, and time of high-altitude exposure that effectively allows reduction of NO levels in cerebral tissue. ${ }^{17,18}$

Hypoxia is a common factor in AMS for people who ascend to high altitudes. Hypoxia induces cerebral vasodilation through some effectors, such as NO and prostacyclins, which, in turn, produce a vasogenic edema in the cerebral circulation, resulting in headache. ${ }^{7}$ The headache itself can cause other symptoms, such as nausea, vomiting, fatigue, and loss of appetite, which constitutes AMS. Several studies have shown that NO may play a pathogenic role in $\mathrm{AMS}^{21}$ because it mediates hypoxia-induced cerebral vasodilation in humans. Moreover, a regional redistribution of cardiac output tends to increase cerebral flow given, at least in part, by a local increase in NO production during hypoxia exposure. ${ }^{22}$ Studies blocking NO production by use of $\mathrm{N}^{\mathrm{G}}$-monomethyl-L-arginine demonstrated an increase in the cerebral vascular resistance, suggesting an important role for NO production. Furthermore, NO can increase the permeability of the blood-brain barrier. ${ }^{23}$ In addition, L-arginine diet supplementation as a substrate for the NO synthase enzyme increased NO and vascular endothelial growth factor production without producing a beneficial effect in the prophylactic treatment of AMS. ${ }^{24}$ In summary, these results could explain a hemodynamic component of NO in inducing headache during AMS. ${ }^{20,22,23}$

With respect to the $G$ biloba treatment, the commercially prepared extract is virtually identical in its ingredients to the commonly used Ginkgo preparation, EGb761, described by other authors in protocols for 
Table 3. Scores for acute mountain sickness (AMS) symptoms at sea level and high altitude*

\begin{tabular}{|c|c|c|c|}
\hline & Placebo & Acetazolamide & Ginkgo biloba \\
\hline \multicolumn{4}{|l|}{ Sea level symptoms } \\
\hline Headache & $0.14 \pm 0.36$ & $0.11 \pm 0.32$ & $0.11 \pm 0.32$ \\
\hline Gastrointestinal symptoms & $0.17 \pm 0.39$ & $0.08 \pm 0.29$ & $0.08 \pm 0.29$ \\
\hline Fatigue and/or weakness & $0.17 \pm 0.39$ & $0.19 \pm 0.41$ & $0.17 \pm 0.39$ \\
\hline Dizziness/lightheadedness & $0 \pm 0$ & $0.17 \pm 0.39$ & $0.14 \pm 0.36$ \\
\hline Difficulty in sleeping & $0.28 \pm 0.47$ & $0.22 \pm 0.43$ & $0.31 \pm 0.48$ \\
\hline AMS score & $0.75 \pm 0.55$ & $0.78 \pm 0.48$ & $0.81 \pm 0.58$ \\
\hline AMS $(\%)$ & 0 & 0 & 0 \\
\hline \multicolumn{4}{|l|}{ High-altitude symptoms } \\
\hline Headache & $1.28 \pm 0.14 \boldsymbol{\Delta}$ & $0.83 \pm 0.59 \boldsymbol{\Delta} \square$ & $0.19 \pm 0.41 \bullet$ \\
\hline Gastrointestinal symptoms & $0.44 \pm 0.65$ & $0.33 \pm 0.57$ & $0.31 \pm 0.48$ \\
\hline Fatigue and/or weakness & $0.39 \pm 0.52$ & $0.44 \pm 0.51$ & $0.08 \pm 0.23$ \\
\hline Dizziness/lightheadedness & $0.17 \pm 0.38$ & $0.25 \pm 0.5$ & $0.14 \pm 0.36$ \\
\hline Difficulty sleeping & $1.47 \pm 0.74 \boldsymbol{\Delta}$ & $0.83 \pm 0.5 \Delta \boldsymbol{\square}$ & $0.22 \pm 0.43 \bullet$ \\
\hline AMS score & $3.75 \pm 1.79 \boldsymbol{\Delta}$ & $2.47 \pm 1.3 \mathbf{\Delta} \boldsymbol{\square}$ & $0.94 \pm 0.76 \bullet$ \\
\hline AMS $(\%)$ & $56 \boldsymbol{\Delta}$ & $36 \mathbf{\Delta} \square$ & $0 \bullet$ \\
\hline
\end{tabular}

*Mean $\pm \mathrm{SD}$; filled triangles $(\boldsymbol{\Lambda})$ represent significant differences compared with sea level $(P<.05)$; filled squares $(\boldsymbol{\square})$ represent significant differences compared with the placebo group $(P<.05)$; filled circles $(\bullet)$ represent significant differences between the $G$ biloba and acetazolamide groups $(P<.05)$.

high-altitude exposure. ${ }^{15-17}$ Moreover, the EGb761 extract was found to be a NO scavenger in vitro under physiological conditions. ${ }^{12}$ Thus, in humans, nasal-exhaled NO was reduced within 5 days of treatment with $G$ biloba, indicating that $G$ biloba decreases NO production. ${ }^{13}$ These studies suggest that the $G$ biloba treatment needs an initial period, according to our and other results, of at least 24 hours ${ }^{15,16,20,21}$ to decrease the symptoms of AMS by reducing NO production. ${ }^{13}$ The diminishment in AMS symptoms could be caused by a decrease in NO production due to NO synthase inhibition mediated by $G$ biloba, thereby reducing cerebral perfusion and permeability of the blood-brain barrier. ${ }^{24}$ A review by Heo and colleagues ${ }^{25}$ described that vascular endothelial growth factor in high concentrations is capable of destabilizing barrier integrity and altering tight junction proteins in cerebral edema caused by a stroke. Considering the association between NO production and vascular endothelial growth factor, we speculate that the reduction in NO production ameliorates AMS symptoms.

Several studies have associated the role of free radicals in the manifestation of AMS or cerebral edema. ${ }^{25-27}$ One possibility could be that $G$ biloba contains a pow-

Table 4. Cardiorespiratory responses in studied groups*

\begin{tabular}{|c|c|c|c|c|}
\hline & & Placebo & Acetazolamide & Ginkgo biloba \\
\hline \multirow[t]{4}{*}{ Sea level } & $\operatorname{HR}\left(\min ^{-1}\right) \dagger$ & $75 \pm 16$ & $79 \pm 17$ & $79 \pm 17$ \\
\hline & $\mathrm{SAP}(\mathrm{mmHg})$ & $128 \pm 12$ & $127 \pm 13$ & $125 \pm 11$ \\
\hline & $\mathrm{DAP}(\mathrm{mmHg})$ & $75 \pm 9$ & $76 \pm 11$ & $74 \pm 9$ \\
\hline & $\operatorname{SatO}_{2}(\%)$ & $98.1 \pm 1$ & $97.8 \pm 1.2$ & $98.8 \pm 1.5$ \\
\hline \multirow[t]{4}{*}{ High altitude } & $\mathrm{HR}\left(\mathrm{min}^{-1}\right)$ & $90 \pm 15 \boldsymbol{\Delta}$ & $94 \pm 14 \boldsymbol{\Delta}$ & $87 \pm 7 \boldsymbol{\Delta}$ \\
\hline & $\mathrm{SAP}(\mathrm{mmHg})$ & $127 \pm 14$ & $123 \pm 11$ & $128 \pm 9$ \\
\hline & $\mathrm{DAP}(\mathrm{mmHg})$ & $77 \pm 8$ & $77 \pm 8$ & $71 \pm 8$ \\
\hline & $\mathrm{SatO}_{2}(\%)$ & $84 \pm 3 \boldsymbol{\Delta}$ & $89 \pm 2 \Delta \square$ & $92 \pm 2 \boldsymbol{\Delta}$ \\
\hline
\end{tabular}

\footnotetext{
*Mean $\pm \mathrm{SD}$; filled triangles $(\boldsymbol{\Delta})$ represent significant differences compared with sea level $(P<.05)$; filled squares $(\boldsymbol{\square})$ represent significant differences compared with the placebo $(P<.05)$.

$\nmid \mathrm{HR}$ indicates heart rate (beats per minute); SAP indicates systolic arterial pressure (mm Hg); DAP indicates diastolic arterial pressure (mm $\mathrm{Hg}) ; \mathrm{SatO}_{2}$ indicates oxygen saturation.
} 
erful antioxidant that inhibits AMS. However, a study performed by Bayley and colleagues ${ }^{26}$ in humans exposed to hypoxia of $12 \%$ (near $4600 \mathrm{~m}$ ) for 18 hours demonstrated an increase in the brain volume independent of oxidative stress, barrier dysfunction, raised lumbar pressure, or vascular damage. Their results suggest that free-radical-mediated vasogenic edema is not an important pathophysiological event observed in the highaltitude headache. This result contradicts 2 previous studies performed by the same authors. ${ }^{10,28}$ This discrepancy could be explained because these studies included physical activity and rate of ascent, which are established prooxidant stimuli. At high altitude and with exercise, skeletal tissue was more susceptible to oxidative damage and might have further compounded extracellular radical formation. In contrast, the central nervous system can provide a mechanism of neuroprotection by increasing the cerebral blood flow to defend against low-tissue $\mathrm{PO}_{2}$.

On the other hand, the higher oxygen saturation levels observed in the group treated with $G$ biloba could be explained in the context of previous results showing that $G$ biloba inhibited phosphodiesterase action, thus enhancing a vasodilation of the parietal smooth muscle cell ${ }^{12}$ and, in turn, increasing tissue perfusion and decreasing local hypoxia. Furthermore, in rats exposed to hypoxia and treated with $G$ biloba, high-altitude pulmonary edema was reduced possibly by a reduction in hypoxic pulmonary hypertension. ${ }^{14}$ These results could explain the higher oxygen saturation observed in our study. This increase in arterial oxygenation could explain the constant delivery of oxygen to the peripheral tissues, thereby decreasing prooxidant stimuli.

Protocols have been developed to prevent malaise at high altitude. Acetazolamide is commonly used to prevent AMS symptoms in tourists, trekkers, or climbers who are exposed to high altitude. In the past, higher doses were used, but currently lower doses are adequate to prevent high-altitude illness. ${ }^{3}$ Our results observed in participants treated with acetazolamide are similar to those found in other studies. ${ }^{7,8,20}$ According to information reported in the literature, acetazolamide prevents AMS by increasing the ventilatory drive given by metabolic acidosis. ${ }^{6}$ Furthermore, studies on cerebral blood flow in humans have shown that acute administration of $1 \mathrm{~g}$ acetazolamide by intravenous injection or a single oral administration of the same dose produced a $20 \%$ to $30 \%$ increase in cerebral blood flow. ${ }^{29,30}$ However, during prolonged treatment with acetazolamide, the increase in cerebral blood flow was maintained for only 2 hours and thereafter decreased. ${ }^{29}$ This augmentation in cerebral blood flow is not mediated by $\mathrm{NO}$ because $\mathrm{NG}_{-}$ monomethyl-L-arginine blockade had no effect on the increase of cerebral blood flow. ${ }^{30}$ In summary, the increase in cerebral perfusion and ventilation that has been associated with high arterial oxygen saturation in connection with metabolic acidosis could reduce cerebral hypoxia, thereby reducing reactive oxygen species or NO production, and this could explain its effect on the diminishment of AMS symptoms.

Two limitations of the present study must be considered: the small number of participants included in our study and the fact that our study was not blinded. It will be important to evaluate the efficacy of $G$ biloba in preventing AMS at higher altitudes $(4000+\mathrm{m})$ and in a double-blinded fashion.

\section{Conclusions}

Prophylactic treatment with $G$ biloba (doses of $80 \mathrm{mg} /$ $12 \mathrm{~h}$ given at $24 \mathrm{~h}$ before high-altitude ascension and with continued treatment for 3 days at high altitude) reduced the symptoms of AMS and increased arterial oxygen saturation in a population without previous highaltitude exposure.

\section{Acknowledgments}

We thank the Mayor of Ollagüe, Carlos Reygadas, for providing all needed facilities to perform our studies, and we are also grateful for the financial support from Grant PEI-1332 Project given by the Investigation Unit at the University of Antofagasta, Antofagasta, Chile.

\section{References}

1. Gilbert DL. The first documented description of mountain sickness: The Andean or Pariacaca story. Respir Physiol. 1983;52:327-347.

2. Ward MP, Milledge JS, West JB. Acute and subacute mountain sickness. In: High Altitude Medicine and Physiology. London: Chapman \& Hall Medical; 1995:366-387.

3. Hackett PH, Rennie D, Levine HD. The incidence, importance, and prophylaxis of acute mountain sickness. Lancet. 1976;2:1149-1155.

4. Moraga FA, Osorio J, Vargas M. Acute mountain sickness in children at 3500 m. Wilderness Environ Med. 2002;13: 51-54.

5. Vargas M, Osorio J, Jiménez D, Moraga FA, Hudson C, Cortés G. Mal agudo de montaña a 3500 y 4250 m: Un estudio de incidencia y severidad de sintomatología. $R e$ vísta Médica de Chile. 2001;129:166-172.

6. Swenson ER, Leatham KL, Roach RC, Schoene RB, Mills WJ, Hackett PH. Renal carbonic anhydrase inhibition reduces high altitude sleep periodic breathing. Respir Physiol. 1991;86:333-343.

7. Hackett PH, Roach R. High-altitude illness. N Engl J Med. 2001;345:107-114. 
8. Serra J, Barriento C, Esnaola C, Bravo J, Cifuentes F, Moraga FA. Chachacoma ¿Un tratamiento alternativo para la prevención del mal agudo de montaña? Revista Ciencia y Salud. 2001;5:36-42.

9. Galarza M, Peñalosa R, Echalar L, Aguilar M, Spielvoguel H, Sauvain M. Efectos del acullico de coca en la prueba de tolerancia a la glucosa. Medicina. 1997;57:261-264.

10. Bailey DM, Davies B. Acute mountain sickness: prophylactic benefits of antioxidant vitamin supplementation at high altitude. High Alt Med Biol. 2001;2:21-29.

11. Kleijnen J, Knipschild P. Ginkgo biloba. Lancet. 1992; 340:1136-1139.

12. Marcocci L, Maguire JJ, Dry-Lefaix MT, Packer L. The nitric oxide-scavenging properties of Ginkgo biloba extract E Ginkgo biloba761. Biochem Biophys Res Commun. 1994;201:748-755.

13. Jowers C, Shih R, James J, Deloughery TG, Holden WE. Effects of Ginkgo biloba on exhaled nitric oxide during normobaric hypoxic in humans. High Alt Med Biol. 2004; 54:445-449.

14. Berg JT. Ginkgo biloba extract prevents high altitude pulmonary edema in rats. High Alt Med Biol. 2004;5:429434.

15. Roncin JP, Scwartz F, D'Arbigny PE. Ginkgo biloba761 in placebo of acute mountain sickness and vascular reactivity to cold exposure. Aviat Space Environ Med. 1996;67:445452.

16. Gertsch JH, Seto TB, Mor J, Onopa J. Ginkgo biloba for the prevention of severe acute mountain sickness (AMS) starting one days before rapid ascent. High Alt Med Biol. 2002;3:29-37.

17. Gertsch JH, Basnyat B, Johnson EW, Onopa J, Holck PS. Randomised, double blind, placebo controlled comparison of Ginkgo biloba and acetazolamide for prevention of acute mountain sickness among Himalayan trekkers: the prevention of high altitude illness trial (PHAIT). $\mathrm{Br}$ Med J. 2004;328:797-801.

18. Chow T, Browne V, Heileson HK, Wallace F, Anholm J, Green SM. Ginkgo biloba and acetazolamide prophylaxis for acute mountain sickness: a randomized, placebo-controlled trial. Arch Intern Med. 2005;165:296-301.

19. Lake-Louise consensus on the definition and quantification of high altitude illness. In: Sutton JR, Coates G, Houston
CS, eds. Hypoxia and Mountain Medicine. Burlington, VT: Queen City Printers Inc; 1992:327-330.

20. West J. Human tolerance to extreme altitudes. In: LeónVelarde F, Arregui A, eds. Hipoxia: Investigaciones Básicas y Clínicas. Lima, IFEA-UPCH; 1993:227-241.

21. Roach RC, Hackett PH. Frontiers of hypoxia research: acute mountain sickness. J Exp Biol. 2001;204:31613170 .

22. Leadbetter G, Maakestad K, Olson S, Hackett PH. Ginkgo biloba reduces incidence and severity of acute mountain sickness. High Alt Med Biol. 2001;2:110.

23. Van Mil AHM, Spilt A, Van Buchem MA, et al. Nitric oxide mediates hypoxia-induced cerebral vasodilation in humans. J Appl Physiol. 2002;92:962-966.

24. Schilling L, Wahl M. Mediators of cerebral edema. $A d v$ Exp Biol Med. 1999;464:123-141.

25. Mansoor JK, Morrissey BM, Walby WF, et al. L-arginine supplementation enhances exhaled NO, breath condensate VEGF, and headache at 4,342 m. High Alt Med Biol. 2005; 6:289-300.

26. Heo HE, Han SW, Lee SK. Free radicals as triggers of brain edema formation after stroke. Free Radic Biol Med. 2005;39:51-70.

27. Bailey DM, Kleger GR, Holzgraefe M, Ballner PE, Bärtsch P. Pathophysiological significance of perooxidative stress, neuronal damage, and membrane permeability in acute mountain sickness. J Appl Physiol. 2004;96: 1459-1463.

28. Bailey DA, Roukens R, Knauth M, et al. Free radicalmediated damage to barrier function is not associated with altered brain morphology in high-altitude headache. J Cereb Blood Flow Metab. 2005;1-3.

29. Bailey DM, Davies B, Young IS. Intermittent hypoxic training: implications for lipid peroxidation induced by acute normoxic exercise in active men. Clin Sci (Lond). 2001;101:465-475.

30. Friberg L, Kastrup J, Rizzi D, Jensen JB, Lassen NA. Cerebral blood flow and end-tidal $\mathrm{PCO}_{2}$ during prolonged acetazolamide treatment in humans. Am J Physiol. 1990; 258:H954-H959.

31. Kiss B, Dallinger S, Findl O, Rainer G, Eichler $\mathrm{H}$. Schmetterer L. Acetazolamide-induced cerebral and ocular vasodilation in human is independent of nitric oxide. Am J Physiol. 1999;276:R1661-R1667. 